\title{
Using evidence-based dissemination and implementation strategies to improve routine communication between general practitioners and community mental health teams
}

\author{
AIMS AND METHOD \\ To improve the quality of written \\ communication between general \\ practitioners (GPs) and community \\ mental health team (CMHT) members \\ concerning patients newly referred \\ to two inner-city CMHTs. Following a \\ benchmark audit of a random sample \\ of referral and assessment letters, \\ locally agreed good practice \\ protocols were shared widely,
}

accompanied by a dissemination and implementation strategy.

RESULTS

Significant improvements occurred in both GP and CMHT letters; these were most dramatic after 1 year, but tailed off considerably in the second year despite continued efforts to implement the protocols' standards.

\author{
CLINICAL IMPLICATIONS \\ Planned dissemination and \\ implementation strategies can help \\ to improve routine clinical \\ communication between CMHTs and \\ GPs through the use of good practice \\ protocols, thus improving shared \\ working between primary and \\ secondary care providers.
}

The significance and extent of communication difficulties between National Health Service (NHS) primary and secondary care services, particularly in London, are well established (Kerwick et al, 1997). When the project reported here began, these difficulties were clearly reflected in local tensions between service providers. Overstretched community mental health teams (CMHTs) were dissatisfied with high rates of clinic non-attendance among new referrals to the team, and had a negative perception that general practitioners (GPs) involved CMHTs too readily in the care of those with less severe mental health problems. General practitioners, in turn, wanted CMHT members to be more accessible by becoming practice-based. Letters were the most commonly used form of communication between the two, and were frequently regarded as inadequate.

Developing more effective shared care between primary and secondary services is a key feature of the National Service Framework for Mental Health (Department of Health, 1999). Although the acceptability of a protocol-based approach in primary care remains controversial (Kendrick, 2000), guidelines can be effective in influencing professional practice, but only when accompanied by dissemination and implementation strategies (Grimshaw \& Russel, 1993). There has been recent interest in collaborative initiatives to improve the communication between primary and secondary care services (Jankowski, 2001). Strategies for dissemination aim to raise participants' awareness, and evidence-based approaches include the use of 'printed educational materials' (Freemantle et al, 2000): in our study these materials concisely described the project's rationale, aims and organisational endorsements. Implementation strategies aim to influence participants' decisions and actions: we adopted a 'decision support system' (Hunt, 1998) in the form of laminated A4 desktop 'reminders' for GPs and CMHT members and 'performance feedback'
(Jamtvedt et al, 2003) in the form of 6-monthly audit reports sent to participating practices and CMHTs.

The aim of this project was to explore the effectiveness of using this combination of evidencebased dissemination and implementation strategies to support a locally-developed written communication protocol for GPs and CMHTs, using the relevance and reliability of their routine written communication about newly referred patients over a 2-year period as an outcome measure.

\section{Method}

\section{Setting}

Paddington is a typical inner-city area of London with a total population of 80000 persons, comprising a substantial mix of ethnic groups, and of affluence and deprivation. North and South Paddington CMHTs operate an open referral system from at least 46 referring general practices in the area.

\section{Sample}

Through the provision of link workers, the CMHTs had established connections with the 26 practices (link practices) referring the most new patient cases each year. A random sample of new patient names was selected from all new link practice referrals from the Central and North West London Mental Health Trust's clinical information system, for each period of the study. For each case, the GP's referral letter was traced from the patient's file in the CMHT records library. The same method was used to select an independent, random sample of $\mathrm{CMHT}$ letters for patients participating in a first assessment at any time over the preceding 12 months. 


\section{Developing the protocols}

The project team comprised a project worker (T.W.) and psychiatrist (S.M.) working with an advisory group made up of a local GP, a community psychiatric nurse and a CMHT administrator. The important components of written communication between primary and secondary care services were agreed by consensus and provided a provisional framework of information items for both GPs' referral letters (Box 1) and CMHTs' new assessment letters (Box 2). Following discussion between the project team and a wider group of local GPs at their postgraduate educational meeting and with members of the participating CMHTs at their weekly team meetings, the final protocols were agreed.

\section{Strategies for dissemination and implementation}

At the start of the project, copies of the protocols and accompanying information were sent by post to all participating link practice GPs and CMHT members, together with a desktop decision support tool that would act as a reminder of the relevant items to be included in clinical letters. Updates were sent out at 6 -monthly intervals throughout the project. Performance feedback (Jamtvedt et al, 2003) was provided by posting baseline and successive audit results to participating practices, at the start of the project and at 6-monthly intervals thereafter. In addition, CMHT link workers were asked to discuss the protocols in detail at the practice meetings they attended, and if possible with individual members of the primary health care team.

Box 1. Quality criteria for general practitioners referral letters to the community mental health team

- Presenting problem

- Past psychiatric history

- Current social circumstances

- Current psychotropic medication

- Patient's view of the referral

- Statement about risk and urgency

Box 2. Quality criteria for community mental health teams' new patient assessment letters to the referring general practitioner

- Provisional diagnosis

- Prognosis

- Treatment plan - psychological interventions

- Treatment plan - pharmacological interventions

- Treatment plan - psychosocial interventions

- Named principal mental health worker (care coordinator)

- Details of next appointment

\section{Developing the audit tool}

Protocol criteria provided the basis of an audit tool designed to detect whether audited letters contained the recommended information items. To improve the tool's reliability, it was piloted on a small sample $(n=10)$ by the two members of the project team independently before it was finalised.

\section{Data collection}

Data were gathered from the letters held in patient files in the patient services library at the CMHTs for three separate periods: 'time 1 ' was the 12-month period prior to the project starting (these data served as a baseline measure); 'time 2', and 'time 3 ' were consecutive 12 -month periods during which the project ran.

\section{Data analysis}

Data were entered onto an Access database spreadsheet, summated and expressed as means, and analysed using the Statistical Package for the Social Sciences, version 10. Non-parametric data were analysed using the KruskalWallis and $\chi$-squared tests.

\section{Results}

General practitioners were the source of the largest number of referrals, with the next largest number coming from the local accident and emergency department. Participating (link) practices accounted for around half of the GP referrals during each study period. The proportion of these patients who completed an assessment with the $\mathrm{CMHT}$ increased significantly over the course of the study (Table 1).

\section{Quality of referral letters}

At the end of time 2, there was a trend towards improved performance for all six GP referral letter criteria compared with the baseline standard. At time 3, performance had deteriorated in comparison with time 2 . During the course of the project, improved performance was statistically significant for four of the six criteria (Fig. 1).

\section{Quality of CMHT letters}

At time 2, CMHT letters showed improvement on all seven criteria compared with the baseline measures. At time 3, further improvement was evident on five of seven criteria and there was marginal deterioration on two criteria. Overall, performance had improved significantly on five of seven criteria since the quality initiative began (Fig. 2).

\section{Reliability of correspondence}

The completeness of CMHT clinical records was used as a proxy measure for the reliability of correspondence exchanged between practitioners. The percentage of original papers 
Table 1. Comparison of all referral categories, general practitioner referrals and link practice referrals, completed CMHT new assessments and shared case-load size over the three study periods

original papers

\begin{tabular}{llll} 
& $1997-1998$ & $1998-1999$ & $1999-2000$ \\
\hline Total annual referrals to Paddington CMHTs: $n$ & 1056 & 945 & 996 \\
Annual GP referrals: $n$ (\% of total) & $661(63)$ & $550(58)$ & $614(62)^{1}$ \\
Annual link practice referrals: $n$ (\% of all GP referrals) & $556(53)$ & $417(44)$ & $476(48)^{1}$ \\
Link practice new referrals completing CMHT assessment: & $369(66)$ & $276(66)$ & $423(89)^{2}$ \\
$\quad n$ (\% of link practice referrals) & & &
\end{tabular}

$\mathrm{CMHT}$, community mental health team; GP, general practitioner.

1. Not significant.

2. $P>0.001\left(\chi^{2}=84\right)$

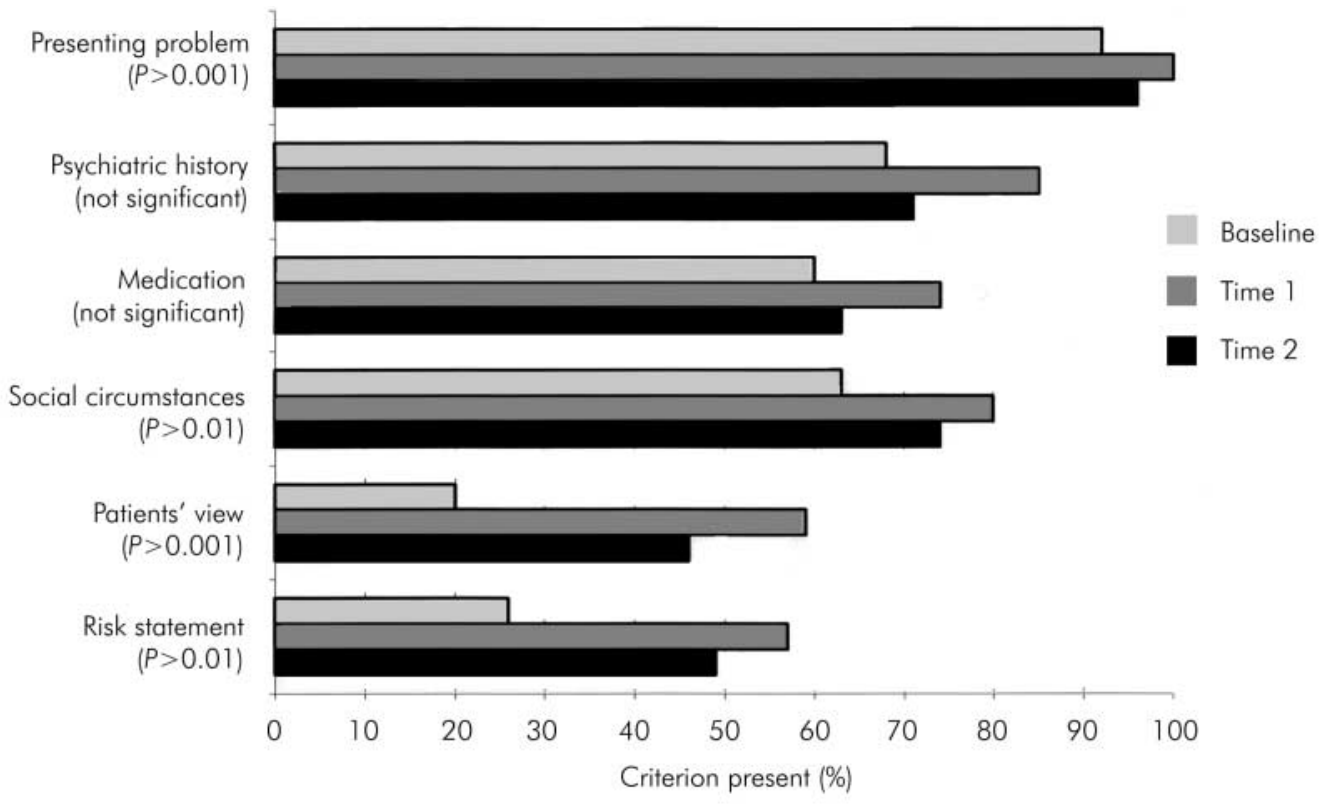

Fig. 1. Compliance of general practitioner referral letters with six quality criteria at 3-yearly intervals.

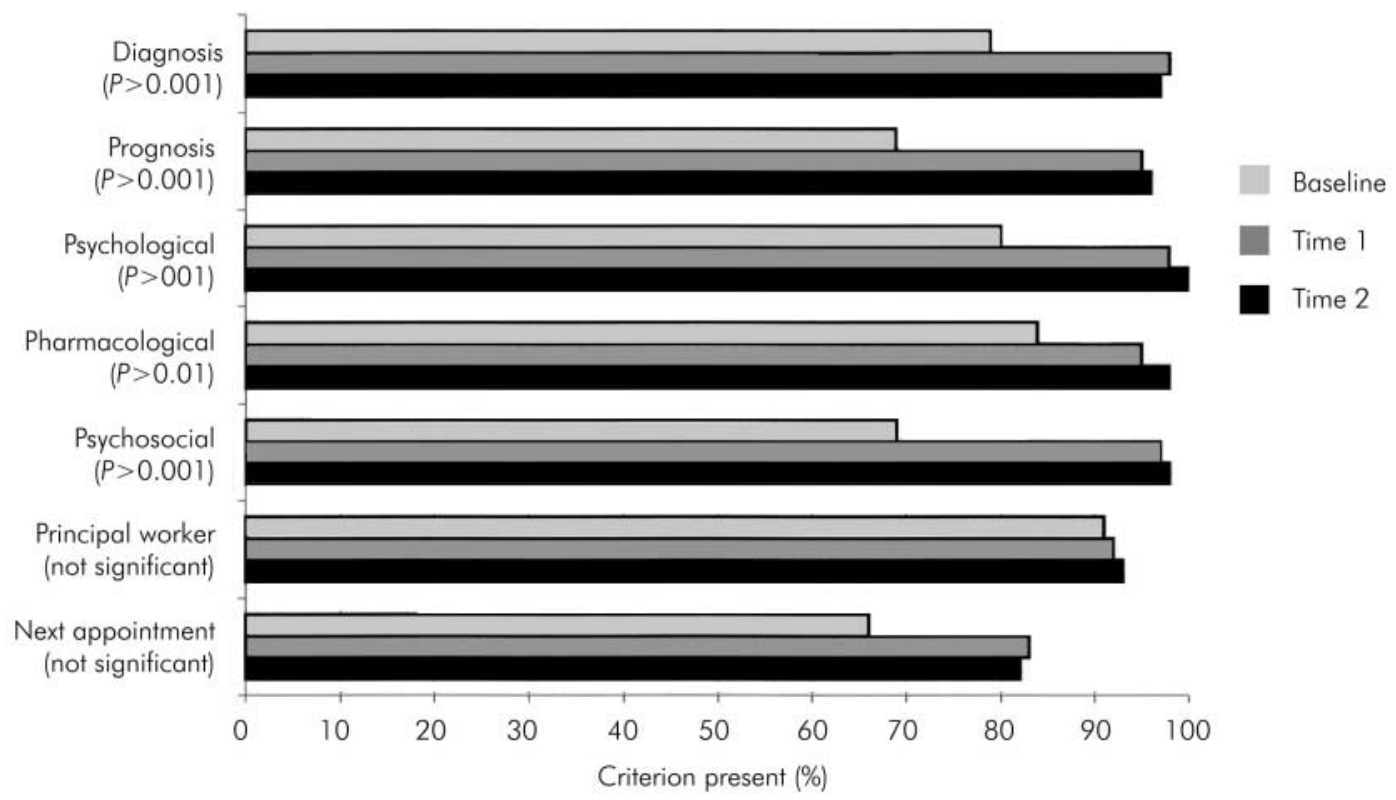

Fig. 2. Compliance of community mental health team letters with seven quality criteria at 3-yearly intervals. 
Table 2. Comparison of sample size and number of letters traced for general practitioner and community mental health team correspondence

\begin{tabular}{|c|c|c|c|}
\hline & Time 1 & Time 2 & Time $3^{1}$ \\
\hline \multicolumn{4}{|l|}{ GP letters } \\
\hline Sample size: $n$ & 80 & 78 & $260^{1}$ \\
\hline Letters retrieved: $n(\%)$ & $58(72)$ & $58(74)$ & $227(87)$ \\
\hline \multicolumn{4}{|l|}{ CMHT letters } \\
\hline Sample size: $n$ & 80 & 78 & 85 \\
\hline Letters retrieved: $n(\%)$ & $58(72)$ & $62(79)$ & 72 (85) \\
\hline
\end{tabular}

CMHT, community mental health team; GP, general practitioner.

1. In the third period of the study the introduction of feedback comparing individual practices' performance with the average performance of practices across the sample required a three-fold increase in the size of the GP sample. New referral data held on the information system were sorted by practice and ten randomly selected new referrals (or all the previous year's referrals if there were fewer than ten) were selected from each practice's list.

assessments accompanied by routine correspondence from the CMHT to the GP increased progressively during the project, but by time 3 no CMHT correspondence to the GP could be traced in $15 \%$ of cases. Although all GP referrals were made in writing, by the final year no GP letter could be traced in $13 \%$ of cases. Taken together, these findings suggest that either the administrative procedures supporting the clinical communication task, or the reliability with which the clinical task was performed, or a combination of the two, remained unreliable (Table 2).

\section{Discussion}

A combination of evidence-based dissemination and implementation strategies improved the quality of both GP and CMHT letters significantly during the project, and audit of administrative procedures suggested that reliability had also improved. Using and maintaining a combination of dissemination and implementation tools is more effective (Oxman, 1994) but, we discovered, also requires considerably more organisational skills than using one type of tool alone, particularly when large numbers of participants are involved. The extent of change achieved in the first year, considering the number of GPs and $\mathrm{CMHT}$ members we involved in the audit, lends support to the effectiveness of using more than one evidence-based implementation or dissemination tool to promote change in practitioners' behaviour and decision-making.
By the end of the second year, as the 'halo effect' of the audit began to dim, a considerable proportion of letters still fell below the desired standards of quality and reliability; some gains made early on in the project had been lost, and administrative procedures remained unsatisfactory. We concluded that achieving more 'seamless' shared working between primary and secondary care will require much more than a protocolbased approach across the interface of the existing organisation of services towards more integrated working styles, models of care and treatment settings for GPs and CMHT practitioners.

\section{Acknowledgements}

The authors want to thank Bharti Rao for her statistical advice in preparing this paper, and Professor Peter Tyrer for his comments on an earlier draft.

\section{Declaration of interest}

None.

\section{References}

DEPARTMENT OF HEALTH (1999) National Service Framework for Mental Health Standards and Service Models. London: DoH.

professional practice and health care outcomes (Cochrane Review). InThe Cochrane Library, Issue 4. Chichester: JohnWiley and Sons.

FREEMANTLE, N., HARVEY, E. L., WOLF， JANKOWSKI, R. F. (2001) Implementing F., et al (2000) Printed educational material: effects on professional practice and health care outcomes. Cochrane Database of Systematic Reviews, Cochrane Library, Issue 2 Oxford: Update Software.

GRIMSHAW, J. M. \& RUSSEL, I.T. (1993) Effect of clinical guidelines on medical practice: a systematic review of rigorous evaluations. Lancet, 342 1317-1322.

HUNT, D. L. (1998) Effects of computerbased clinical decision support systems on physician performance and patient outcomes: a systematic review. JAMA, 280, 1339-1346

JAMTVEDT, G., YOUNG, J. M., KRISTOFFERSON, D. T., et al (2003) Audit and feedback: effects on national guidelines at a local level. BMJ, 322, 1258-1259.

KENDRICK, T. (2000) Why can't GPS follow guidelines on depression? BMJ, 320, 200-201.

KERWICK, S., TYLEE, A. \& GOLDBERG, D. (1997) Mental health services in primary care in London. In: London's Mental Health: A Report for the King's Fund London Commission (eds S. Johnson, R. Ramsay \& G. Thornicroft), pp. 131-142. London: King's Fund. OXMAN, A. (1994) No magic bullets: a systematic review of 102 trials of interventions to help health care professionals deliver services more effectively or efficiently. London: North EastThames Regional Health Authority.

Tracy White previously Senior Project Worker, LInC (Liaison at the Interface of Care Project, * *Sarah Marriott Consultant Psychiatrist, Paterson Centre for Mental Health, Central and North West London Mental Health Trust, 20 South Wharf Road, LondonW2 $1 \mathrm{EE}$ original papers 